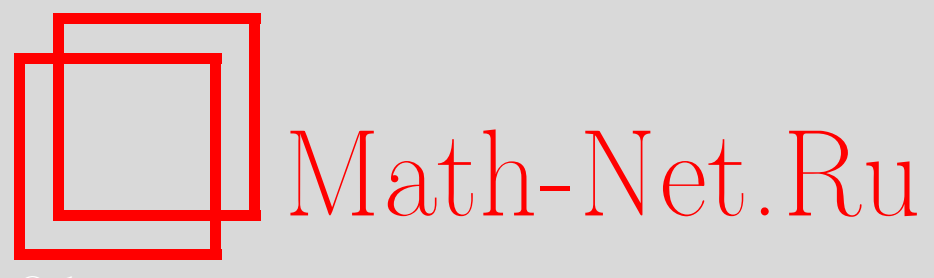

С. Ю. Доброхотов, С. Я. Секерж-Зенькович, Один класс точных алгебраических локализованных решений многомерного волнового уравнения, Матем. заметки, 2010, том 88, выпуск 6, 942-945

DOI: https://doi.org/10.4213/mzm8916

Использование Общероссийского математического портала Math-Net.Ru подразумевает, что вы прочитали и согласны с пользовательским соглашением http: //www . mathnet.ru/rus/agreement

Параметры загрузки:

IP: 54.174 .149 .18

26 апреля 2023 г., $12: 17: 36$

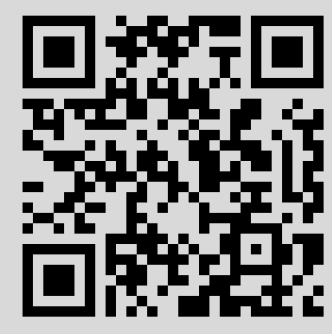




\section{Один класс точных алгебраических локализованных решений многомерного волнового уравнения}

\section{С. Ю. Доброхотов, С. Я. Секерж-Зенькович}

1. Введение. В работах [1]-[3] получены асимптотические решения задачи Коши для линейных гиперболических систем (с переменными коэффициентами), включая и волновое уравнение, в том случае когда начальные условия локализованы в окрестности точки и имеют вид функции $V(x / \mu)$, где $V(y)$ - гладкая функция, убывающая при $|y| \rightarrow \infty$ быстрее $1 /|y|^{1+\delta}, \delta>0, \mu>0$ - малый параметр, описывающий "степень локализации". В этих работах было показано, что с асимптотической точки зрения в таких задачах имеется явление метаморфозы профиля решения, аналогичное явлению метаморфозы разрывов в теории обобщенных решений гиперболических систем [4]: при $t=0$ решение локализовано в окрестности точки, а при $t \gg \mu$ решение локализовано в окрестности замкнутой гиперповерхности - волнового фронта. С точки зрения квазиклассических асимптотик точка $x=0$ оказывается фокальной точкой и глобальный ответ выражается в виде обобщенного канонического оператора Маслова, реализация которого приводит к различным формулам в различных областях пространства. В этой заметке мы приводим широкий класс некоторых точных алгебраических решений задачи Коши для многомерного волнового уравнения

$$
\frac{\partial^{2} u}{\partial t^{2}}-c^{2} \sum_{j=1}^{j=n} \frac{\partial^{2} u}{\partial x_{j}^{2}}=0, \quad x \in \mathbb{R}^{n},\left.\quad u\right|_{t=0}=V(x),\left.\quad u_{t}\right|_{t=0}=0 .
$$

Приводимые решения основаны на начальных условиях рассмотренных (в двумерном случае) в работах [5], [6], более того, в [5], [6] одно из таких решений построено в параметрической форме (см. также [7], [8]). Наше представление кажется нам более простым и эффективным. Построенные решения иллюстрируют в многомерном случае указанное явление "метаморфозы профиля решения". Разумеется, это явление хорошо известно в математической и теоретической физике, и, на наш взгляд, любопытный ("методологический”) факт здесь состоит в том, что оно описывается элементарными алгебраическими функциями, что позволяет явным образом проследить весь процесс метаморфозы решения для волновых уравнений в любой размерности. По всей видимости, рассматриваемый в этой заметке пример с этой точки зрения является уникальным.

2. “Основное решение”. Сначала выберем функции $V(x)$ в следующем виде. Положим

$$
V(x)=\frac{A}{\left(1+|x|^{2} / \mu^{2}\right)^{(n+1) / 2}} .
$$

Здесь $A, \mu$ - вещественные константы, $\mu>0$.

УтвержДЕние 1. Решение задачи Коши (1), (2) определяется формулой

$$
u(x, t)=A \operatorname{Re} \frac{(1+i c t / \mu)}{\left((1+i c t / \mu)^{2}+|x|^{2} / \mu^{2}\right)^{(n+1) / 2}} .
$$

ДокАЗАТЕЛЬство легко получается простым дифференцированием. Более "идейное" доказательство опирается на тот факт, что преобразование Фурье $\widetilde{V}(k), k=\left(k_{1}, \ldots, k_{n}\right)-$ двойственные переменные по отношению к $x=\left(x_{1}, \ldots, x_{n}\right)$, функции $(2)$ равно $A e^{-\mu|k|}$.

Работа выполнена при поддержке Российского фонда фундаментальных исследований (грант № 08-01-00726). 
Последующее применение метода Фурье к задаче (1),(2) приводит к явно вычисляемым интегралам, дающим формулу (3).

3. “Операторы рождения и возмущенные решения". Очевидно дифференцирование любого решения задачи (1) по любой переменной $x_{j}$ дает новое решение волнового уравнения. В этом смысле операторы $\partial / \partial x_{j}$ играют роль операторов рождения для (1). Эти элементарные соображения приводят к следующему очевидному утверждению. Пусть $Q(k)$ - некоторый полином аргументов $k=\left(k_{1}, \ldots, k_{n}\right)$. Ему соответствует дифференциальный оператор $\widehat{Q}=Q(\mu \nabla)$, где $\nabla$ - оператор градиента.

УтВеРЖДЕНИЕ 2. Функиии

$$
u_{Q}(x, t)=A \operatorname{Re}\left(Q(\mu \nabla) \frac{(1+i c t / \mu)}{\left((1+i c t / \mu)^{2}+|x|^{2} / \mu^{2}\right)^{(n+1) / 2}}\right),
$$

являются решением задачи Коши (1) с

$$
V_{Q}(x)=\widehat{Q} \frac{A}{\left(1+|x|^{2} / \mu^{2}\right)^{(n+1) / 2}} .
$$

4. Поведение функций $u(x, t)$ и $u_{Q}(x, t)$. Рассмотрим случай, когда $\mu$ мало. Тогда при $t=0$ эти функции локализованы в $\mu$-окрестности точки $x=0$. При $t \gg \mu / c$ эти функции локализованы в окрестности сферы $S_{t}=\{|x|=c t\}$ (что естественно), и асимптотика функции $u(x, t)$ определяется следующим образом. Введем сферические координаты $\psi=\psi_{1}, \ldots, \psi_{n-1}$ (углы) и $r=|x|$, и в окрестности $S_{t}$ будем использовать координаты $\psi=\psi_{1}, \ldots, \psi_{n-1}$ и $y=r-c t$. Единичный вектор с углами $\psi=\psi_{1}, \ldots, \psi_{n-1}$ будем обозначать $\mathbf{n}(\psi)$. Тогда асимптотики $u(x, t)$ и $u_{R}(x, t)$ при $t \gg \mu / c$ определяются формулами

$$
\begin{aligned}
u(x, t) & =\frac{\mu^{(n-1) / 2}}{2^{(n+1) / 2}(c t)^{(n-1) / 2}} \operatorname{Re} \frac{i}{(i+y / \mu)^{(n+1) / 2}}+O\left(\mu^{(n+1) / 2}\right), \\
u_{Q}(x, t) & =\frac{\mu^{(n-1) / 2}}{2^{(n+1) / 2}(c t)^{(n-1) / 2}} Q\left(\mu \mathbf{n}(\psi) \frac{\partial}{\partial y}\right) \operatorname{Re} \frac{i}{(i+y / \mu)^{(n+1) / 2}}+O\left(\mu^{(n+1) / 2}\right) .
\end{aligned}
$$

При соответствующей нормировке при $\mu \rightarrow+0$ функция $u(x, 0)$ в пределе переходит в $\delta$-функцию Дирака $\delta(x)$, а при $t>\varepsilon>0$ - в $\delta$-функцию на сфере $|x|=c t$, и тем самым переходит в хорошо известное в математической физике обобщенное (фундаментальное) решение многомерного волнового уравнения с $\delta$-функцией в начальных условиях [9]. Ясно, что асимптотика по параметру $\mu$ эквивалентна асимптотике по времени $t$ при $t \gg \mu$ (для любых $\mu$, в частности при $\mu=1$ ). Функция $u(x, t)$, разумеется, сферически симметрична, а функции $u_{Q}(x, t)$, вообще говоря, нет. Производные по переменным $x_{j}$ в точной формуле и по переменной $y$ в асимптотической формуле создают осцилляции у решений в окрестности фронта, но также могут и менять амплитуду в тех точках, где значение полинома $Q$ на векторе $\mathbf{n}(\psi)$ оказывается малым, и определять, таким образом, “диаграмму направленности" волны. Например, в двумерном случае в координатах $(y=|x|-c t, \psi)$, $\psi$ - полярный угол, имеем

$$
x=(c t+y)\left(\begin{array}{c}
\cos \psi \\
\sin \psi
\end{array}\right),
$$

и если $\widehat{Q}=\partial^{m} / \partial x_{1}^{m}$, то с точностью до $O\left(\mu^{(n+1) / 2}\right)$

$$
u_{Q}(x, t) \approx \cos ^{m} \psi \frac{\mu^{(n-1) / 2}(-1)^{m}(n+1)(n+3) \cdots(n+1+2 m)}{2^{(n+1) / 2+m}(c t)^{(n-1) / 2}} \operatorname{Re} \frac{i}{(i+y / \mu)^{(n+1) / 2+m}} .
$$

Осцилляции в направлении оси $x_{1}$ создают "начальный импульс" у волны, при этом по-прежнему фронт волны определяется уравнением $|x|=c t$, но амплитуда становится очень маленькой в окрестности точек $\psi= \pm \pi / 2$. 
Приведем несколько рисунков, иллюстрирующих поведение функций $u$ и $u_{R}$ для двумерного случая, $\mu=0.2, c=1$. На рис. 1-3 приведены графики "основной" функции и времен $t=0, t=0.1, t=0.8$.
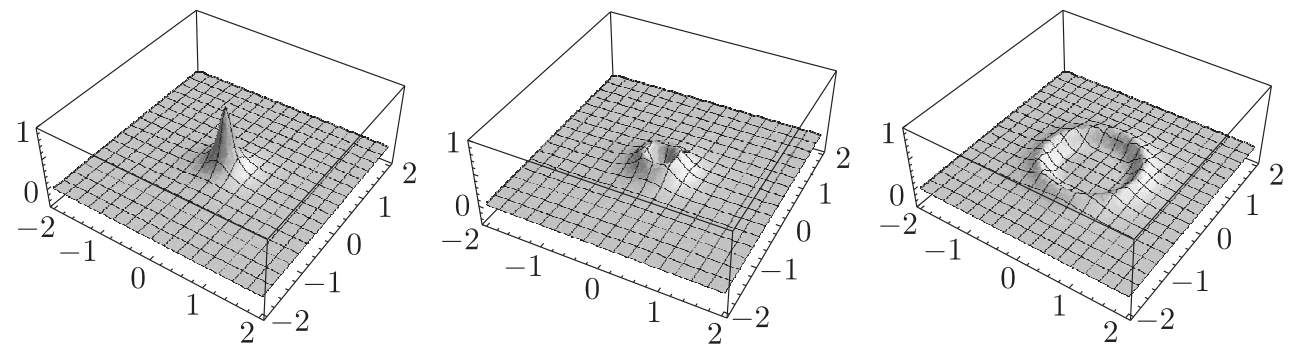

Рис. 1. Графики “основной" функции в моменты времени $t=0, t=1.2, t=4$

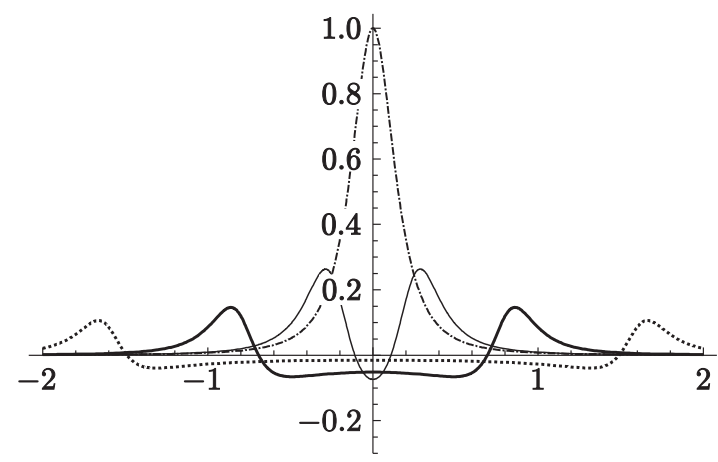

Рис. 2. Профили "основной" функции в моменты времени $t=0, t=1.2$, $t=4, t=8$
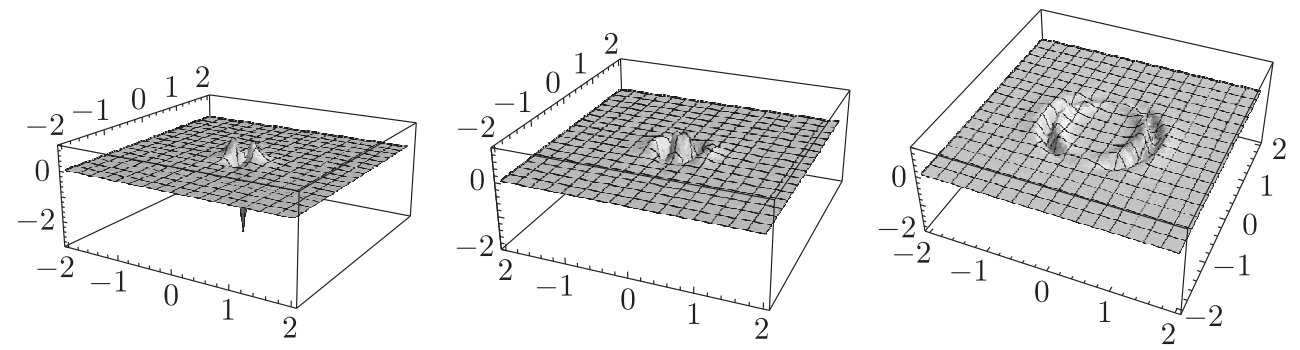

Рис. 3. Графики второй производной по переменной $x_{1}$ в моменты времени $t=0, t=1.2, t=4$

На рис. 3 в те же моменты времени приведены графики возбужденной функции оператором $\partial^{2} / \partial x_{1}^{2}$, а на рис. 4 приведены профили этой функции для полярных углов $\psi=0$, $\psi=\pi / 4, \psi=\pi / 2$ в моменты времени $t=0, t=1.2, t=4, t=8$. Видно, что при $\psi=\pi / 2$ амплитуда решения быстро исчезает со временем.

В заключение отметим, что константу $A$ в формуле (2), как и коэффициенты полинома $Q$, можно выбрать комплексными, построенные функции, по-прежнему, будут 

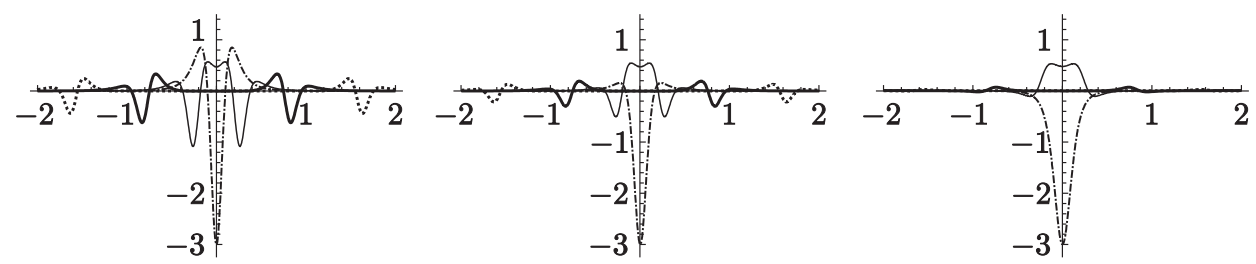

Рис. 4. Профили функции второй производной по переменной $x_{1}$ для значений полярных углов $\psi_{1}=0$ (левый рисунок), $\psi_{1}=\pi / 4$ (средний рисунок), $\psi_{1}=\pi / 4$, (правый рисунок) в моменты времени $t=0, t=1.2$, $t=4$.

определять точные решения волнового уравнения, но начальные условия, разумеется, будут другими.

Мы благодарим С. И. Безродных и Е. Н. Пелиновского за полезные замечания.

\section{СПИСОК ЦИТИРОВАННОЙ ЛИТЕРАТУРЫ}

[1] S. Yu. Dobrokhotov, A. I. Shafarevich, B. Tirozzi, Russ. J. Math. Phys., 15:2 (2008), 192-221. [2] С. Ю. Доброхотов, Б. И. Волков, С.Я. Секерж-Зенькович, Б. Тироцци, Фунд. прикл. геофизика, 2, 2009, 15-29. [3] Б.И. Волков, С.Я. Секерж-Зенькович, Изв. РАН. МЖГ, 2009, №6, 116-122. [4] В. М. Бабич, Уч. зап. ЛГУ. Сер. матем., 246:32 (1958), 228-260. [5] С. Ф. Доценко, Б. Ю. Сергиевский, Л. В. Черкасов, Исследования иунами, Вып. 1, МГК АН СССР, М., 1986, 7-14. [6] S. Wang, ASCE Specialty Conference on Coastal Hydrodynamics (June 29-July 1, 1987), University of Delaware, 1987, 657-670. [7] Е. Н. Пелиновский, Гидродинамика волн иунами, ИПФ РАН, Нижний Новгород, 1996. [8] S. Ya. Sekerzh-Zenkovich, Russ. J. Math. Phys., 16:2 (2009), 315-322. [9] В. С. Владимиров, Уравнения математической физики, Наука, М., 1981.

\section{С. Ю. Доброхотов}

Институт проблем механики им. А. Ю. Ишлинского РАН,

Московский физико-технический институт

(государственный университет)

E-mail: dobr@ipmnet.ru

\section{С. Я. Секерж-Зенькович}

Институт проблем механики им. А. Ю. Ишлинского РАН, Московский физико-технический институт (государственный университет)

E-mail: seker@ipmnet.ru 